Reprod. Nutr. Dévelop., 1986, 26 (2 B), 551-561.

\title{
Relationships between structure and function of lactogenic hormones
}

\author{
Nicole CHÊNE, P. de la LLOSA*, G. CHARPIGNY, J. MARTAL \\ Unité d'Endocrinologie de l'Embryon, Physiologie animale, \\ I.N.R.A., 78350 Jouy-en-Josas, France. \\ * Laboratoire des Hormones Polypeptidiques, \\ C.N.R.S., 91190 Gif-sur-Yvette, France.
}

Summary. Lactogenic activity of several hormone derivatives obtained by chemical modifications of lysine residues was studied by radioreceptor assay.

The relationships between structure and binding to lactogenic receptors are discussed taking into account lysine residue positions liable to be involved in the location of lactogenic function.

\section{Introduction.}

Placental hormones (PL) or chorionic somatomammotropins (CS) and hypophyseal hormones such as prolactins ( $P R L$ ) or some growth hormones $(G H)$ exhibit lactogenic activity in radioreceptor assay (RRA); they belong to two families of polypeptides with closely related structures probably resulting from a common ancestral short peptide (Niall et al., 1971).

In some ruminants, PL hormones also exhibit a growth hormone activity (Chan et al., 1976 ; Martal and Djiane, 1977). In human female, hCS is unable to bind to growth hormone receptors (Tsushima and Friesen, 1973), while hGH is endowed with both somatotropic and lactogenic action. Besides, amino-acid sequences of hGH, hCS and oPRL are well known (Niall et al., 1971 ; Li et al., 1971). Conversely, the oCS primary structure has not yet been elucidated, though the physicochemical characterization has been performed by Handwerger et al. (1974), Martal and Djiane (1975), Chan et al. (1976). For all these reasons, these four molecules are very interesting compounds for studying relationships between chemical structure and biological activity.

In the present work, we studied the effects of some chemical changes in lysine residues of these four hormones upon the binding capacity to lactogenic receptors. Only chemical changes preserving the basicity of the group (methylation, ethylation, guanidination and acetiminidation) were achieved, preventing proteins from secondary effects on their conformation.

Afterwards, a comparison was made between the already known primary structures of these hormones in relationship to their lactogenic activity. 


\section{Material and methods.}

\section{Hormones.}

oCS was purified in our laboratory and was not subjected to any contamination from either GH or PRL as reported by Martal (1978).

Global aminoacid composition was established by means of a Technicon autoanalyser. Lysine content was 14 residues for a MW of 22000 daltons.

Ovine prolactin was a purified preparation kindly provided by NIH (PS-7).

hGH was a gift from Drs F. Dray and F. Groh (Institut Pasteur, Paris).

hCS was provided by USB Corporation.

\section{Chemical modifications.}

Four chemical modifications were performed. Methylation was accompanied by a slight change in the $\mathrm{pK}$. Ethylation introduced a longer alkyl chain. Guanidination and acetiminidation modified the $\mathrm{pK}$ of the protein and the distance between the positive charge of the protein and the polypeptide backbone.

Reductive alkylation. - Reductive methylation and ethylation were carried out by addition of sodium borohydride and formaldehyde or acetaldehyde to the hormones $(0.5-1.0 \mathrm{mg})$ dissolved in borate buffer $(\mathrm{pH} \mathrm{9)}$ as described by Means and Feeney (1968). The solutions were dialyzed against a pyridine solution (1\%) and freeze-dried. Determinations of lysine, methyl- and ethylysine were made using a Technicon autoanalyser and elution gradients containing isopropanol (Means and Feeney, 1968 ; de la Llosa et al., 1974). A blank for biological assays was prepared by treating hormone with borohydride and borate buffer $\mathrm{pH} \mathrm{9,} \mathrm{no}$ added aldehyde). In the case of ovine prolactin whose disulfide bridges are particularly labile to the reductive action of borohydride $\left(\mathrm{BH}_{4}\right)^{-}$, the reductive alkylation was performed at lower concentration of $\left(\mathrm{BH}_{4}\right)^{-}(20 \mathrm{mM})$, for a shorter period $(30 \mathrm{~min})$ and in the presence of iodoacetamide $(40 \mathrm{mM})$ to block the $\mathrm{SH}$ groups avoiding the disordered reconstitution of disulfide bonds.

Guanidination. - This reaction was performed using $0.3 \mathrm{M} \mathrm{0}$-methyl-isourea sulfate (Aldrich, France) at $\mathrm{pH} 10.3$ and $5^{\circ} \mathrm{C}(0.7 \mathrm{mg}$ hormone $/ 0.15 \mathrm{ml})$ for $24 \mathrm{~h}$. At the end of the reaction, the solutions were dialyzed against pyridine solutions. After one day of reaction, some of the solutions became slightly opalescent. In both cases, the solutions were centrifuged and the precipitate discarded. The degree of guanidination was measured by aminoacid analysis. A control was prepared by treatment of the hormone at $\mathrm{pH} 10.4$ for one day at $5{ }^{\circ} \mathrm{C}$.

Acetimidination. - Prolactin $(0.7 \mathrm{mg} / 0.5 \mathrm{ml})$ was treated by $1 \mathrm{M}$ ethylacetimidate hydrochloride (Aldrich) for $24 \mathrm{~h}$ at $\mathrm{pH} 10.3$ and $5^{\circ} \mathrm{C}$. hGH was treated in the same conditions but at a much lower concentration of reagent $(0.01 \mathrm{M})$ to obtain a small degree of chemical modification. Determination of the $\epsilon$-acetimidyllysine was performed by aminoacid analysis (Plapp and Kim, 1974).

Radioreceptor assay of lactogenic activity. - This activity was measured as described by Martal and Djiane (1975). 
Mammary gland membranes were obtained from rabbits treated on Day 10 of lactation with $2-\alpha$-bromocryptine (CB 154, Sandoz, $2 \mathrm{mg}$ twice daily for 2 days) to desaturate their receptors. The standard curve was established by incubation of membrane receptors, radioiodinated prolactin and different concentrations of unlabelled ovine prolactin (NIH-PS7, $24 \mathrm{IU} / \mathrm{mg}$ ) for $5 \mathrm{~h}$ at $21^{\circ} \mathrm{C}$. Lactogenic activity of modified hormones was determined by adding these hormone derivatives to the incubation medium instead of the unlabelled prolactin. The specificity of the assay was checked : only hormones with lactogenic activity in the rabbit (prolactins of different species, placental lactogens and human growth hormone) are able to compete with ovine prolactin on the rabbit mammary receptor sites. The rabbit receptor exhibits less strict specificity than the ovine receptor.

\section{Results and discussion.}

Table 1 lists the relative potencies of modified hormones compared to the native hormone (in \%).

A decrease in the lactogenic activity of oCS was observed after treatment with only $\left(\mathrm{BH}_{4}\right)^{-}$as shown by Chêne et al. (1984). This might be due to the disturbance in the conformation caused by partial reduction of disulfide bridges.

This effect could be avoided by adding iodoacetamide reagent in the reductive step as it was done for oPRL (fig. 1, Table 1).

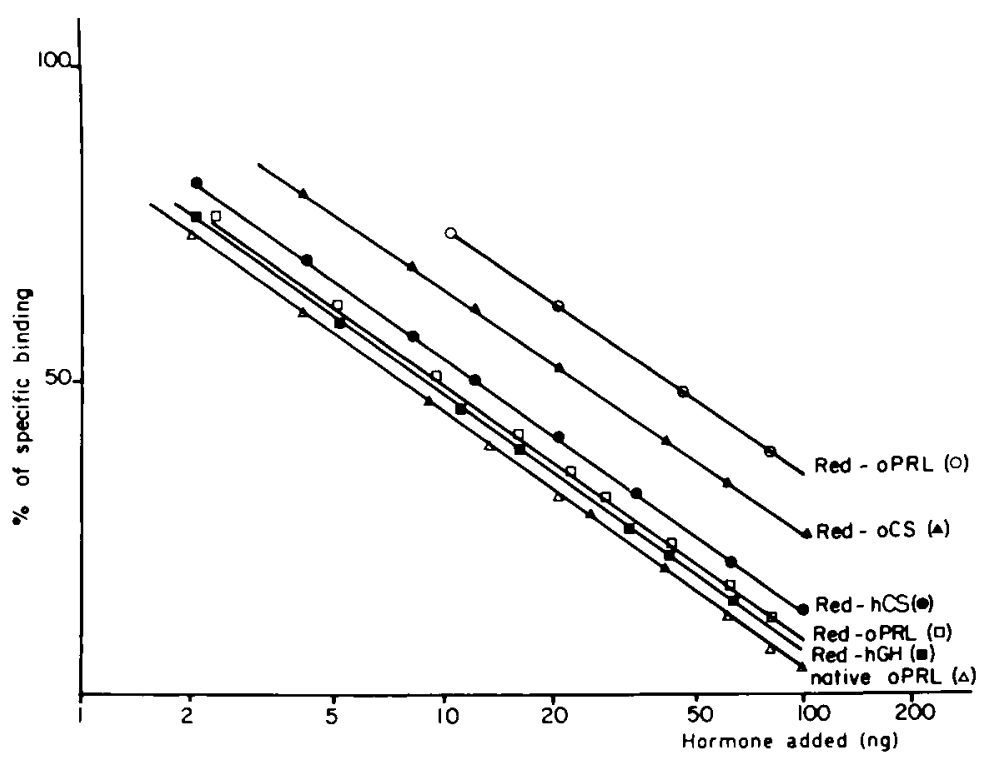

FIG. 1. - Specfic binding of reduced hormones in a radioreceptor assay. reduced oPRL (treated by $\mathrm{BH}_{4}-$ without iodoacetamide) reduced oPRL (treated by $\mathrm{BH}_{4}{ }^{-}$in the presence of iodoacetamide) 
TABLE 1

Compared analysis of the influence of chemical modifications of lactogenic hormones upon their biological activity.

\begin{tabular}{|c|c|c|c|c|c|c|}
\hline $\begin{array}{c}\text { Modified } \\
\text { hormones }\end{array}$ & Reduction & Methylation & Ethylation & $\begin{array}{l}\text { Control } \\
\text { at } \mathrm{pH} 10\end{array}$ & $\begin{array}{l}\text { Guanidina- } \\
\text { tion }\end{array}$ & $\begin{array}{l}\text { Acetimini- } \\
\text { dation }\end{array}$ \\
\hline $\begin{array}{l}\operatorname{OPRL}(9 L)^{*} \\
\text { hCS }(9 L)^{*} \\
\text { hGH }(9 L)^{*} \\
\text { oCS }(14 L)^{*}\end{array}$ & $\begin{array}{c}90 \%(1)(O L)^{* *} \\
83 \%(2)(O L) \\
90 \%\left(^{2}\right)(O L) \\
45.5 \%\left({ }^{2}\right)(O L)\end{array}$ & 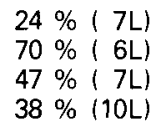 & $\begin{array}{lll}73 & \% & (4 L) \\
80 & \% & (2 L) \\
75 & \% & (5 L) \\
10 \% & (8 L)\end{array}$ & $\begin{array}{l}100 \%(O L) \\
100 \%(O L) \\
100 \%(O L) \\
100 \%(O L)\end{array}$ & $\begin{array}{rlr}21 & \% & (6 L) \\
0 & \% & (7 L) \\
67 & \% & (5 L) \\
1 & \% & (11 L)\end{array}$ & $\begin{array}{c}20 \%(6 L) \\
27 \%(4-5 L) \\
100 \%(1-2 L) \\
29 \%(3 L)\end{array}$ \\
\hline
\end{tabular}

* Number of total lysine residues; ** Number of modified lysine residues ; (1) Protected disulfide bonds ; $\left.{ }^{2}\right)$ No protected disulfide bonds.

In figure 2, methylation seemed very perturbing especially for oPRL. In this reaction, hCS preserved a good activity whereas hGH lost $50 \%$ of its activity.

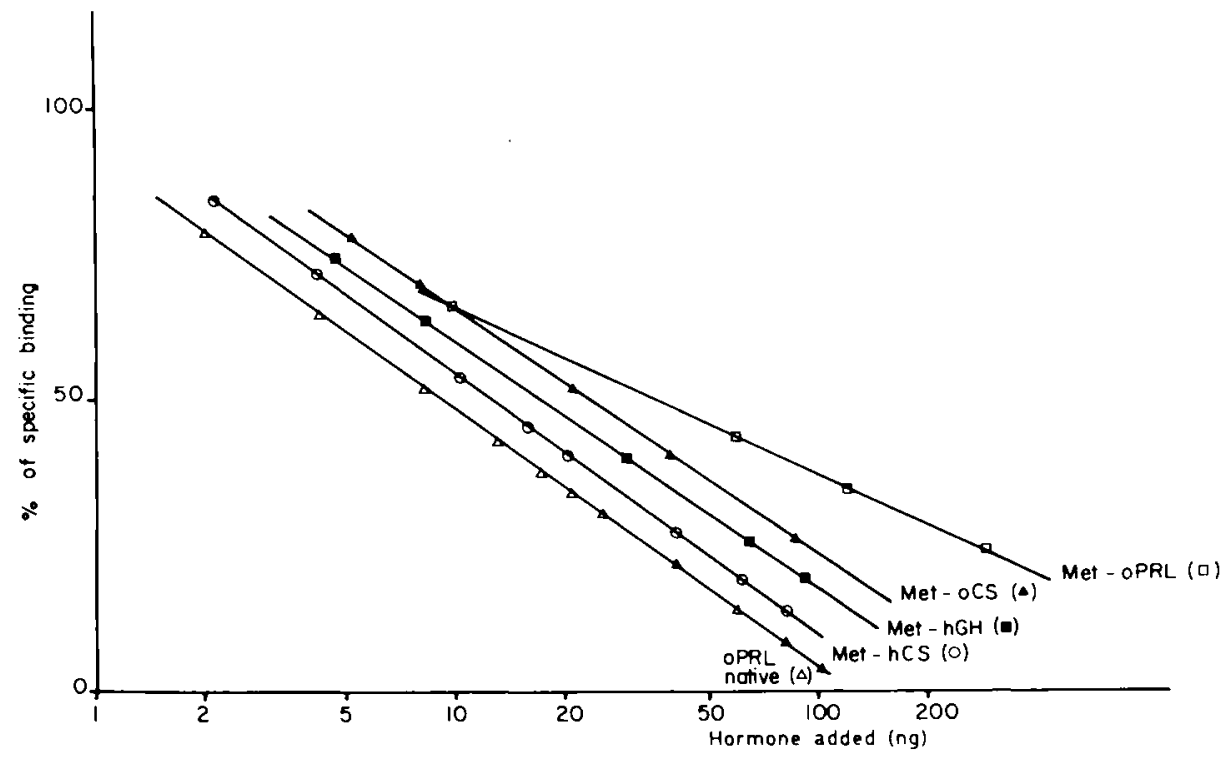

FIG. 2. - Specific binding of methylated hormones in a radioreceptor assay.

In figure 3, ethylation only slightly affected the binding activity of oPRL, $\mathrm{hCS}, \mathrm{hGH}$, but the degree of modification was smaller than that due to methylation. Only oCS exhibited a markedly reduced capacity.

A 5-day treatment of the four hormones at $\mathrm{pH} 10.4$ and at $4{ }^{\circ} \mathrm{C}$ (necessary for guanidination and acetiminidation) did not apparently affect their biological activity.

Lactogenic activity of guanidylated compounds was largely depressed (except for guanidyl-hGH), those of hCS and oCS were completely abolished (see fig. 4, table 1). 


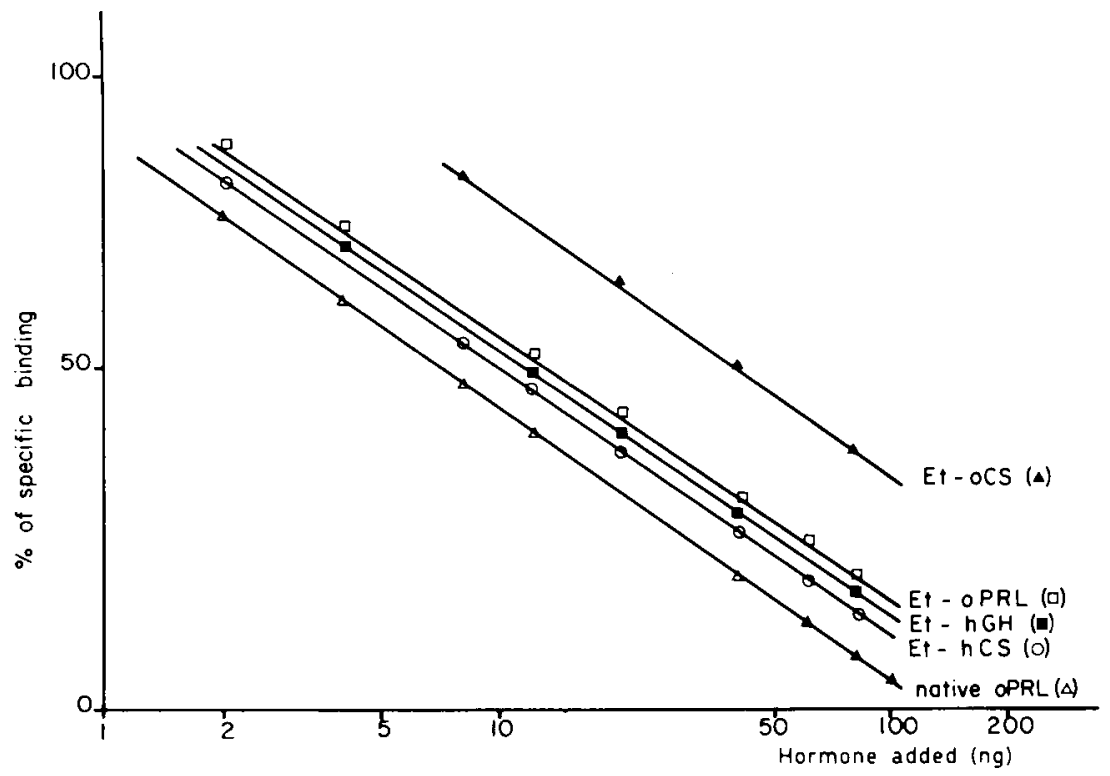

FIG. 3. - Specific binding of ethylated hormones in a radioreceptor assay.

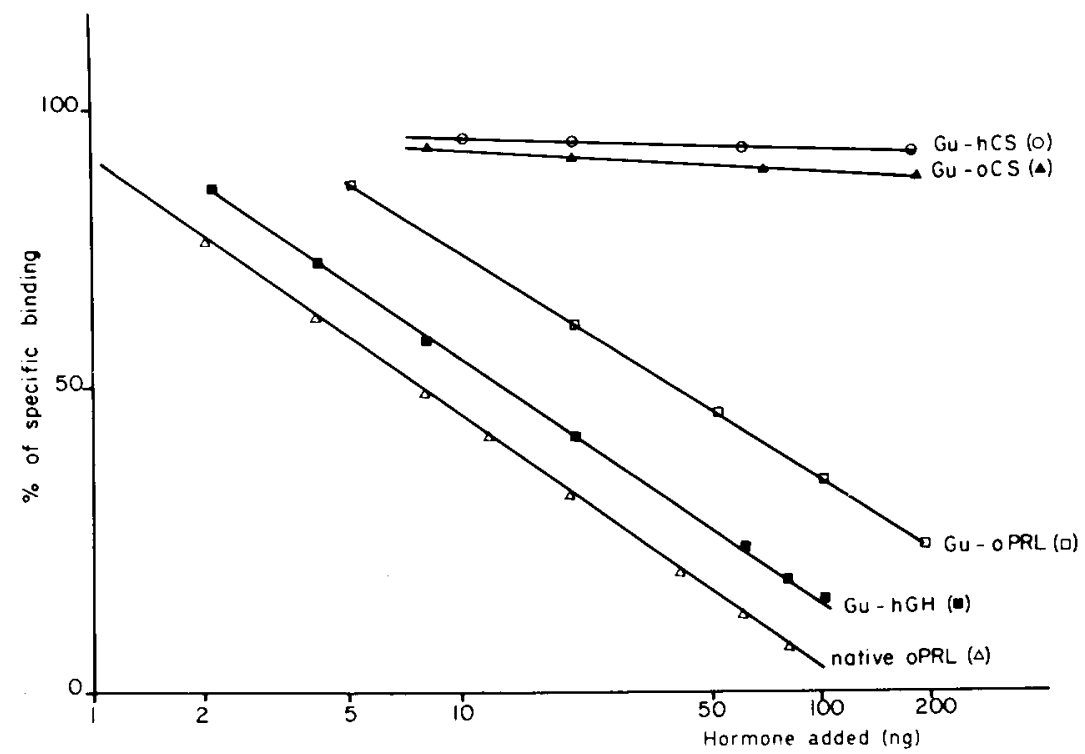

FIG. 4. - Specific binding of guanidinated hormones in a radioreceptor assay.

In figure 5, acetiminidation led to a significant loss of activity for three hormones, only hGH molecule exhibited full activity.

Whatever the type and degree of modification, it may be assumed that lysine residues are involved in the binding capacity of these four hormones to lactogenic 
receptors. A similar conclusion was obtained when studying somatotropic activity of oCS (Chêne et al., 1984) and hGH (Martal et al., 1985).

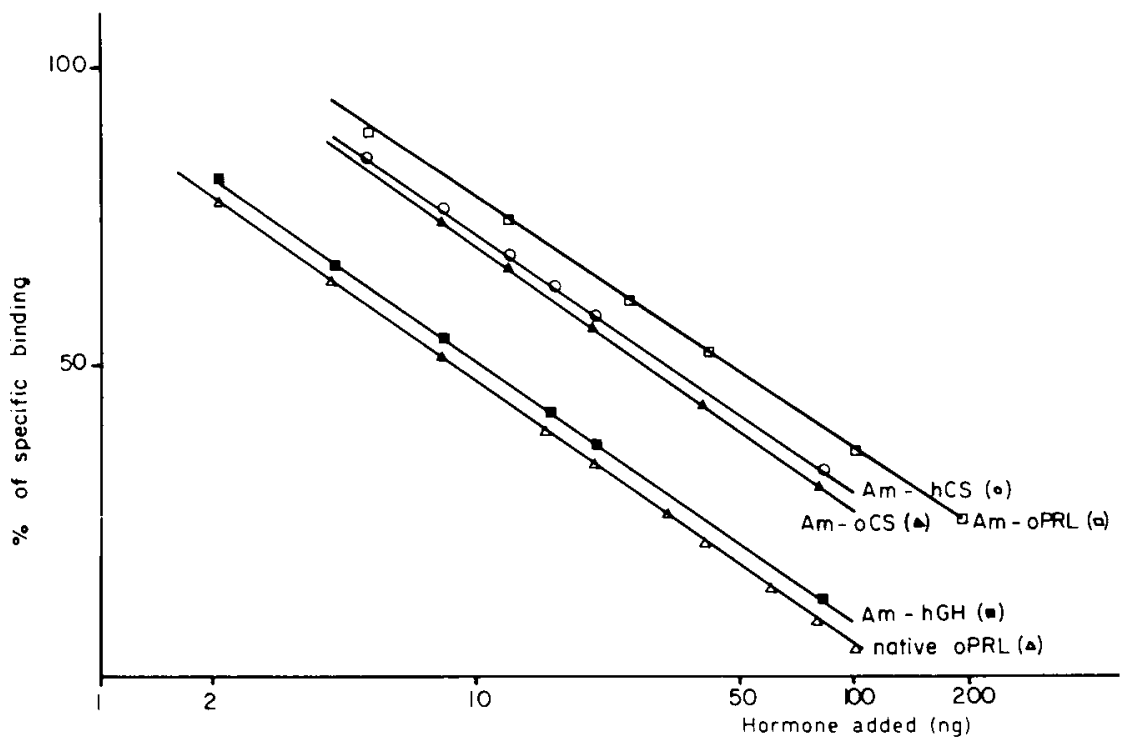

FIG. 5. - Specific binding of acetimidylated hormones in a radioreceptor assay.

Such modifications of the activity are depending on the protein structure, and the examination of aminoacid sequences of these different hormones in relation with lactogenic activity is envisaged in the present paper.

Primary structures of ovine (Li et al., 1970), bovine (Wallis, 1974), porcine (Li, 1976), human (Shome and Parlow, 1977) and mouse PRL (Kohmoto et al., 1984) were elucidated by protein sequencing. The primary sequences of rat (Cooke et al., 1980) and human PRL (Cooke et al., 1981) were recently investigated by complementary DNA sequencing.

Li (1978) established the primary structure of both hCS and hGH molecules. They found $85 \%$ of identical aminoacids and $96 \%$ homology as regards conservative mutations.

In table 2, we compared the aligned sequences of seven hormones : five prolactin hormones, hCS and hGH, according to the methods of Dayhoff (1972), and Martal (1980), taking gaps into account until aminoacids in position 62 ; afterwards disulfide bond between cysteins 62 and 180 imposes a rigid conformation to the molecule, $\mathrm{N}$-terminal gaps being not involved. Only the position of lysine residues and sometimes arginine residues (a basic aminoacid) was considered. Some lysine residues were particularly well preserved during evolution : lysines 128 and 187, they are common to all prolactins (rat, pig, sheep, cattle, man), to hCS - the only placental lactogen whose primary structure was reported - and to all known growth hormones, such as human (Bewley et al., 1972), ovine (Li et al., 1972), pig (Mills and Wilhelmi, 1972), bovine (Wallis, 1973), rat (Wallis and Davies, 1976) and equine GH (Daurat-Larroque et al., 1977). 


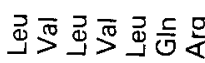

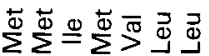

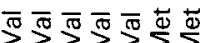

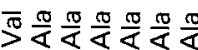

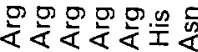

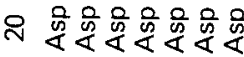

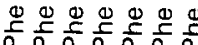

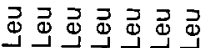

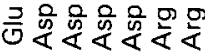

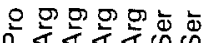

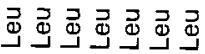

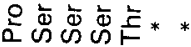

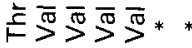
등등듣등단.

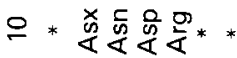

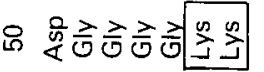
등등등등똗등 등

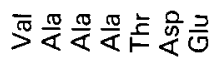
…… * * . . . $\frac{9}{80}$

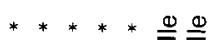

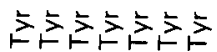

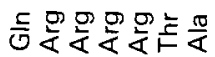

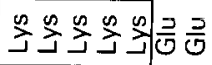

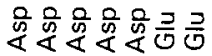

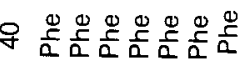

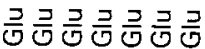

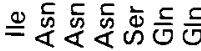

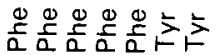

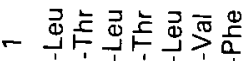

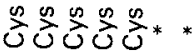

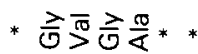

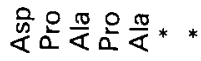
츠ㅇㅡㅡ은추 *

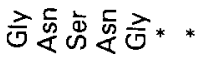

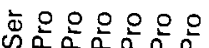

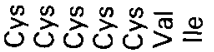

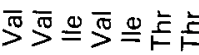

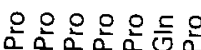
王主王琣主至

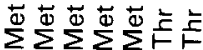

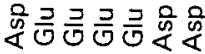

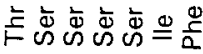

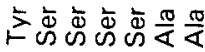

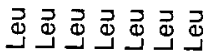

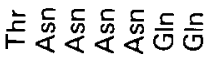

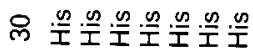

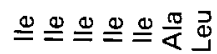

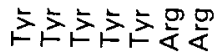

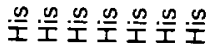

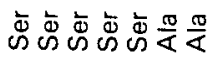

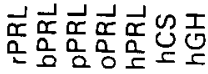

등등등등등긍긍 긍크ㅇㅡㅡㅇ그ㅇㅡㅡㅇ긍긍

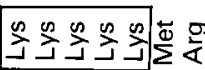

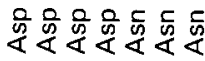
긍크ㅎㅡㅡ근

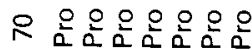

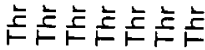

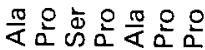

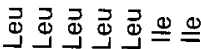

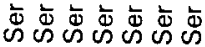

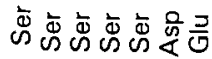

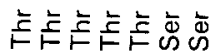

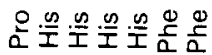

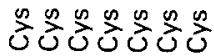
***** * 号寻

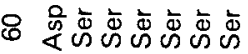

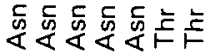

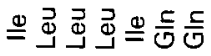

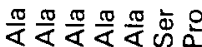

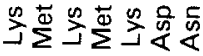

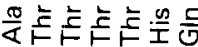

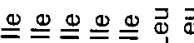

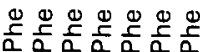

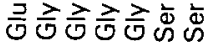

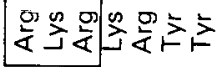

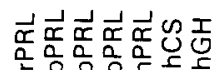

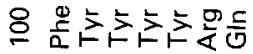

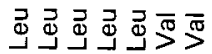
울웅음은웅

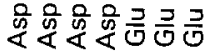

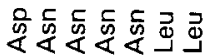

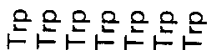

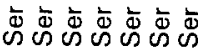

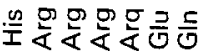

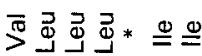

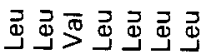

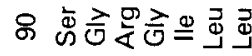

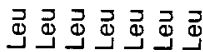

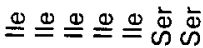

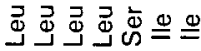

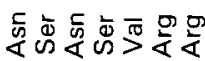

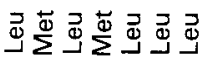

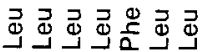

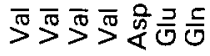

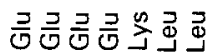

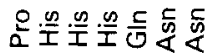

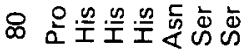

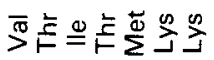
옥등등등등등등 등등등등등등

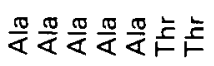

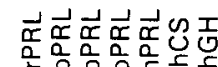




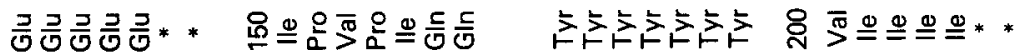

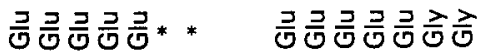

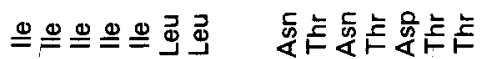

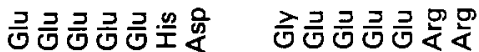

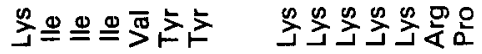

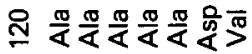

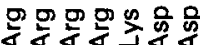

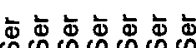

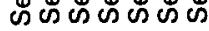

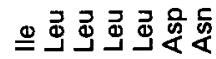

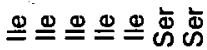

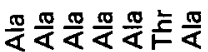

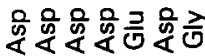

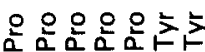

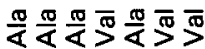

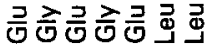

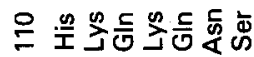
ஹ苍芯芯希员员

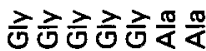

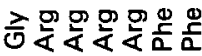
す 증그ㅇㅡㅡㅇㅡㅡ은 * *

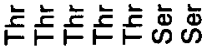

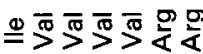

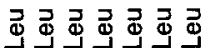

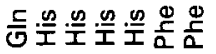

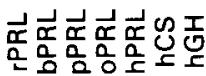

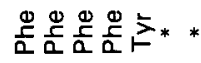

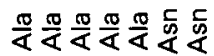

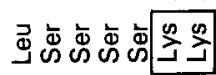

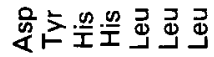

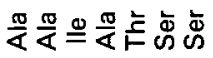

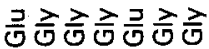

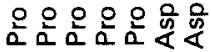

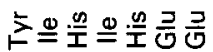

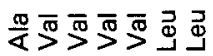

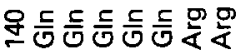

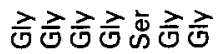

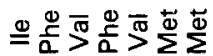

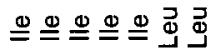

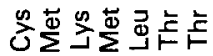
긍 긍 그ㅇㅡㅡㄱㅡㅡ등등

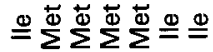

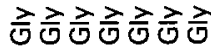
긍긍 킁그ㅇㅡㅡ응긍

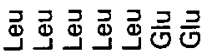

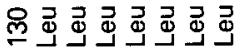

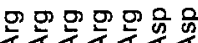

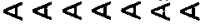

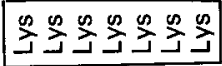

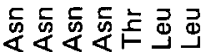

요요

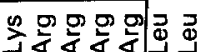

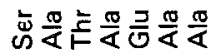

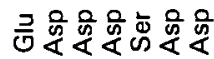
긍긍크ㅇㅡㅡ응오용

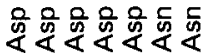

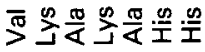

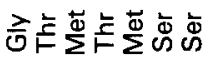

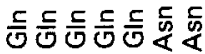

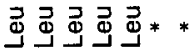

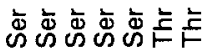

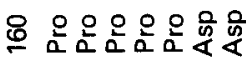

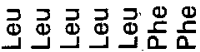

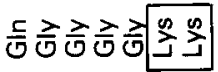

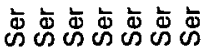

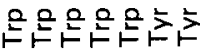

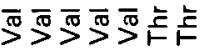

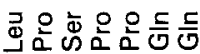

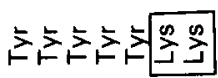
***** 弯焉 $* * * * * \stackrel{\oplus}{\cong}$ 등 그ㅇㅡㅡㄹㅡㅠ 등* *

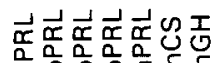

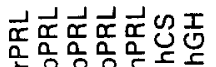

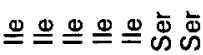

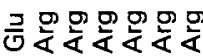

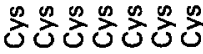

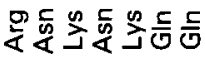

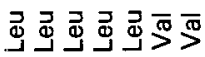

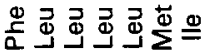

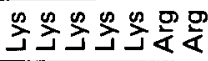

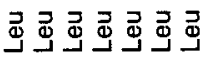

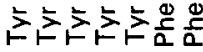

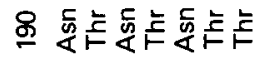

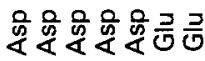

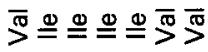

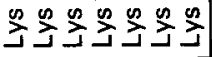

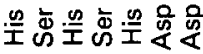

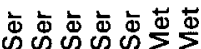

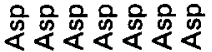

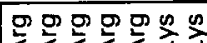

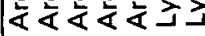

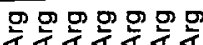

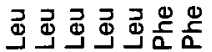
동 造㹡

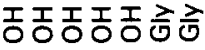

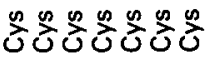
要要要要要离部 委要要要要专京

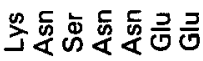

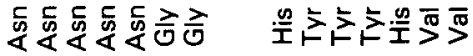

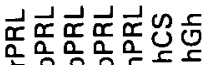

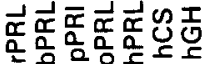


Lysines in position 42 and 51 of PRL family could approximately correspond in $\mathrm{hGH}$ and hCS sequences to the positions of lysine 47 and 50 respectively, which could occupy a similar place in the steric conformation of the molecule when gaps are excluded. Nevertheless, this aminoacid residue 50 of $\mathrm{hGH}$ and hCS was preserved in all known growth hormones, but lysine 47 of hGH and hCS was substituted by a glutamic acid in ovine, bovine, rat and equine $\mathrm{GH}$; these latter hormones are not lactogenic.

Other prolactin lysines $(73,146,193)$ are substituted by arginines in hGH $(73$, $147,193)$ and in hCS $(146,193)$. As residue 73 exists in all the molecules exhibiting prolactin activity except in hCS, this basic residue could contribute to the activity without being indispensable.

Lysines 153 and 158 are common to $\mathrm{hGH}$ and hCS molecules, but they do not exist in PRL family, as arginines 170 and 183 of PRL family are substituted by lysines 172 and 183 in hCS and hGH hormones.

Birk and Li (1978) assumed that an enzymatic cleavage by plasmin of the oPRL peptide bond between Met 56 and Ala 57 destroys biological activity. Lactogenic activity was preserved after selective removal of residues 148-158 by trypsin (table 2), thus lysines 153 and 158 are not implicated in the lactogenic function (Graf et al., 1982).

Graf and Li (1974) and Doneen (1975) showed that partial lactogenic activity was developed by fragment 1-147 of hGH (table 2) suggesting that lysines in position after 147 are not indispensable.

In conclusion, aminoacid residues of PRL family in positions $42,51,128,146$, in positions $47,50,128,147$ for $\mathrm{hGH}$ and for hCS might be essential for the binding to lactogenic receptors. It must be pointed out however that the presence of a basic residue at positions $50,128,147$ in $\mathrm{hGH}$ is observed also in the case of other growth hormones suggesting that the basic residues at these positions are essential for binding but do not define the specificity of the molecule for lactogenic receptors.

Further studies will be required to locate and define precisely the contribution of these lysines and other aminoacids to the lactogenic activity.

$11^{e}$ Réunion du groupe Développement, I.N.R.A., Montpellier, 22-24 mai 1985.

Acknowledgements. - We thank Mrs M. Roy for her expert technical assistance and Mrs D. Molitano for typing this manuscript. We are grateful to Drs F. Dray and F. Groh for their gift of hGH and to N.I.H. for oPRL.

This work has been financially supported by C.N.R.S., Paris (RCP 080768).

\section{Résumé. Hormones lactogènes : relations structure-fonction.}

L'activité lactogène de plusieurs dérivés hormonaux obtenus après modifications chimiques des résidus lysine a été analysée par dosage radio-hormone-récepteur. Les relations entre la structure des hormones modifiées et la liaison aux récepteurs lactogènes sont discutées en considérant les positions des résidus lysines susceptibles d'être impliqués dans la localisation de la fonction lactogène. 


\section{References}

BEWLEY T. A., DIXON J. S., LI C. H., 1972. Sequence comparison of human pituitary growth hormone, human chorionic somatomammotropin and ovine pituitary growth and lactogenic hormones. Int. J. Peptide Protein Res., 4, 281-287.

BIRK Y., LI C. H., 1978. Two fragments from fibrinolysin digests of ovine prolactin : characterization and recombination to generate full immunoreactivity. Proc. nat. Acad. Sci. USA, 75, 2155-2159.

CHAN J. S.D., ROBERTSON H. A., FRIESEN H. G., 1976. The purification and characterization of ovine placental lactogen. Endocrinology, 98, 65-76.

CHÊNE N., MARTAL J., de la LLOSA P., 1984. Involvement of lysine residues in the binding of ovine chorionic somatomammotropin to lactogenic and somatotropic receptors. FEBS Letters, 166, 352-356.

COOKE N. E., COIT D., WEINER R. I., BAXTER J. D., MARTIAL J. A., 1980. Structure of cloned DNA complementary to rat prolactin messenger RNA. J. biol. Chem., 255, 6502-6510.

COOKE N. E., COIT D., SHINE J., BAXTER J. D., MARTIAL J. A., 1981. Human prolactin cDNA structural analysis and evolutionary comparisons. J. biol. Chem., 256, 4007-4016.

DAURAT-LARROQUE S. T., MOYA PORTUGUEZ M. E., SANTOME J. A., 1977. Reaction of bovine and equine growth hormones with tetranitromethane. Int. J. Peptide Protein Res., 9, $119-128$.

DAYHOFF M. O., ECK R. V., PARK C. M., 1972. A model of evolutionary change in proteins. In Atlas of protein sequence and structure, 5, 89-99, Nat. Biochem. Res. Found., Washing ton.

DONEEN B. A., 1976. Biological activities of mammalian and teleostean prolactins and growth hormones on mouse mammary gland and teleost urinary bladder. Gen. comp. Endocrinol., 30, 34-42.

GRAF L., LI C. H., 1974. On the primary structure of pituitary bovine growth hormone. Biochem. biophys. Res. Commun., 56, 168-176.

GRAF L., LI C. H., JIBSON M. D., 1982. Human somatotropin. Selective removal with trypsin of residues 135-145 from the hormone molecule with no loss of biological activity. J. biol. Chem., 257, 2365-2369.

HANDWERGER S., MAURER W., BARRETT J., HURLEY T., FELLOWS R. E., 1974. Evidence for homology between ovine and human placental lactogens. Endocrinol. Res. Commun., 1, 403413.

KOHMOTO K., TSUNASAWA S., SAKIYAMA F., 1984. Complete aminoacid sequence of mouse prolactin. Eur. J. Biochem., 138, 227-237.

LI C. H., 1976. Studies on pituitary lactogenic hormone. Int. J. Peptide Protein Res., 8, 205-224.

LI C. H., 1978. Noncovalent interaction of the $\mathrm{NH}_{2}$-terminal fragment of human somatotropin with the $\mathrm{COOH}$-terminal fragment of human choriomammotropin to generate growth-promoting activity. Proc. nat. Acad. Sci. USA, 74, 1016-1019.

LI C. H., DIXON J. S., LO T. B., SCHMIDT K. D., PANKOV Y. A., 1970. Studies on pituitary lactogenic hormone. The primary structure of the sheep hormone. Arch. Biochem. Biophys., 141, 705-737.

LI C. H., DIXON J. S., CHUNG D., 1971. Primary structure of the human chorionic somatomammotropin (hCS) molecule. Science, 173, 56-58.

LI C. H., DIXON J. S., GORDON D., KNORR J., 1972. Aminoacid sequence of sheep pituitary growth hormone. Int. J. Peptide Protein Res., 4, 151-153.

LLOSA P. de la, DUROSAY M., TERTRIN-CLARY C., JUTISZ M., 1974. Chemical modification of lysine residues in ovine luteinizing hormone. Effect on biological activity. Biochim. biophys. Acta, 342, 97-104.

MARTAL J., 1978. Placental growth hormone in sheep: purification, properties and variations. Ann. Biol. anim. Biochim. Biophys., 18, 45-51.

MARTAL J., 1980. L'hormone lactogène placentaire ovine. Th. Dr. ès Sci., Paris-Orsay Univ.

MARTAL J., CHÉNE N., de la LLOSA P., 1985. Involvement of lysine residues in the binding of hGH and bGH to somatotropic receptors. FEBS Letters, 180, 295-299. 
MARTAL J., DJIANE J., 1975. Purification of a lactogenic hormone in sheep placenta. Biochem. biophys. Res. Commun., 65, 770-778.

MARTAL J., DJIANE J., 1977. Mammotrophic and growth promoting activies of a placental hormone in sheep. J. Steroid Biochem., 8, 414-417.

MEANS G. E., FEENEY R. E., 1968. Reductive alkylation of amino groups in proteins. Biochemistry, 7, 2192-2201.

MILLS J. B., WILHELMI A. E., 1972. Studies on the primary structure of porcine growth hormone 38-44. In PECILE A., MÜLLER E. E. Growth and growth hormone. Excerpta med. Amsterdam.

NIALL H. D., hOGAN M. L., SAUER R., ROSENBAUM I. Y., GReENWOOD F. C., 1971. Sequences of pituitary and placental lactogenic and growth hormones : evolution from a primordial peptide by gene reduplication. Proc. nat. Acad. Sci. USA, 68, 866-869.

PLAPP B. V., KIM J. C., 1974. Determination of $\varepsilon$-acetiminidyl-lysine in proteins. Anal. Biochem., 62, 291-294.

SHOME B., PARLOW A. F., 1977. Human pituitary prolactin (hPRL) : the entire linear aminoacid sequence. J. clin. Endocrinol. Metab., 45, 1112-1115.

TSUSHIMA T., FRIESEN H. G., 1973. Radioreceptor assay for growth hormone J. clin. Endocrinol. Metab., 37, 334-336.

WALLIS M., 1973. The primary structure of bovine growth hormone. FEBS Letters, 35, 11-14.

WALLIS M., 1974. The primary structure of bovine prolactin. FEBS Letters, 44, 205-208.

WALLIS M., DAVIES R. V., 1976. Studies on the chemistry of bovine and rat growth hormones, 4-13. In PECILE A., MÜLLER E. E. Growth hormones and related peptides, Excerpta med. Amsterdam. 